University of Nebraska - Lincoln

DigitalCommons@University of Nebraska - Lincoln

$10-29-2007$

\title{
Linear Tactile Nanodevice with Resolution on Par with Human Finger
}

\author{
Ravi F. Saraf \\ University of Nebraska-Lincoln, rsaraf2@unl.edu \\ Vivek C. Maheshwari \\ University of Nebraska-Lincoln, vmaheshw@uwaterloo.ca \\ Chieu Nguyen \\ University of Nebraska-Lincoln
}

Follow this and additional works at: https://digitalcommons.unl.edu/cbmesaraf

Part of the Biomechanics and Biotransport Commons

Saraf, Ravi F.; Maheshwari, Vivek C.; and Nguyen, Chieu, "Linear Tactile Nanodevice with Resolution on Par with Human Finger" (2007). Ravi Saraf Publications. 6.

https://digitalcommons.unl.edu/cbmesaraf/6

This Article is brought to you for free and open access by the Chemical and Biomolecular Research Papers -Faculty Authors Series at DigitalCommons@University of Nebraska - Lincoln. It has been accepted for inclusion in Ravi Saraf Publications by an authorized administrator of DigitalCommons@University of Nebraska - Lincoln. 


\title{
Linear Tactile Nanodevice with Resolution on Par with Human Finger
}

Saraf, R.F.

Maheshwari, V.

Nguyen, C.

University of Nebraska-Lincoln

This paper appears in: Optical MEMS and Nanophotonics, 2007 IEEE/LEOS International Conference on Publication Date: Aug. 12 2007-July 162007

Pages: 35 - 36

Location: Hualien, Taiwan

ISBN: 978-1-4244-0641-8

INSPEC Accession Number: 9642957

Digital Object Identifier: 10.1109/OMEMS.2007.4373827

Posted online: 2007-10-29 14:58:08.0

\begin{abstract}
A large area thin-film nanodevice made by self-assembly containing electroluminescent nanoparticles is reported. The $100 \mathrm{~nm}$ thick device on application of potential across the top and bottom surface of the film converts local pressure to light. The intensity of the electroluminescent light is linearly proportional to the applied local compressive stress. By imaging the light, the stress distribution over the area of contact is obtained at resolution on par with human finger.
\end{abstract}

Keywords: Electron Tunneling, Nanoparticle self-assembly, Robotics, Tactile sensor, Thin film device 


\title{
Linear Tactile Nanodevice with Resolution on Par with Human Finger
}

\author{
Ravi F. Saraf*, Vivek Maheshwari, Chieu Nguyen \\ Department of Chemical and Biochemical Engineering \\ University of Nebraska - Lincoln, Lincoln, NE 68516, USA \\ Tel 402472 8284, FAX 402472 6989, E-mail rsaraf@unlnotes.unl.edu
}

\begin{abstract}
A large area thin-film nanodevice made by self-assembly containing electroluminescent nanoparticles is reported. The $\sim 100$ $\mathrm{nm}$ thick device on application of potential across the top and bottom surface of the film converts local pressure to light. The intensity of the electroluminescent light is linearly proportional to the applied local compressive stress. By imaging the light, the stress distribution over the area of contact is obtained at resolution on par with human finger.
\end{abstract}

Keywords: Tactile sensor, Electron Tunneling, Thin film device, Nanoparticle self-assembly, Robotics

\section{INTRODUCTION}

Recently, Bill Gates speculated that after the Personal Computer, a humanoid robot in every home would be the next revolution that would affect our lives intrinsically (1). Excluding taste, among the four basic senses, touch at resolution on par with human finger remains a challenge. Without a high resolution and sensitivity large area tactile device, also sometimes referred to as electronic skin, the humaonoid robot may find it difficult to pour and deliver a glass of water to its master (2), or fold the laundry. The current tactile devices with active area of over $1 \mathrm{~cm}^{2}$ have a resolution of $\sim 1-2 \mathrm{~mm}$ (3) in contrast to human finger that has a resolution of $\sim 40 \mu \mathrm{m}$ (4) allowing us to feel a filament of hair on a smooth surface that has a typical diameter of $\sim 100 \mu \mathrm{m}$. Recently, by self-assembling layers of nanoparticles by simple solution deposition process invented by Decher (5) we fabricated a large area device of thickness $\sim 100 \mathrm{~nm}$ to convert compressive stress distribution or pressure on physical contact to light (6). By imaging the distribution of emitted light on a digital camera a "stress image" can be obtained that resolves the distribution of contact-pressure at a resolution of $\sim 20 \mu \mathrm{m}$ (6). Apart form the resolution on par with human sensitivity at contact pressures of 10-60 KPa that are comparable to the human touch, the approach has two practical advantages: (a) The fabrication process that is essentially sequential dip-coating followed by washing and drying will allow deposition of thin film device on large surface of area comparable to displays. (b) The response of the device, i.e., the intensity of light, is linearly proportional to applied stress. The linear relationship makes the grey-scale of the stress-image quantitatively comparable to the contact-pressure distribution. In this report, we present our observations that indicate why an electron tunneling based device that is intrinsically non-linear leads to highly linear response.

\section{DEVICE CHARACTERISTCS}

The $\sim 100 \mathrm{~nm}$ thick nanodevice is a film (Fig. 1) consisting of alternating layers of $\mathrm{Au}$ (10 $\mathrm{nm}$ diameter) and $\mathrm{CdS}$ (3 nm diameter) nanoparticles separated by dielectric layers (DL), composed of stacked alternating layers of poly(styrene sulfonate) (PSS) and poly(allylamine hydrochloride) (PAH) (6). The 2.5 by $2.5 \mathrm{~cm}$ device is made by layer-by-layer selfassembly process (5) on a sputter deposited $\sim 400 \mathrm{~nm}$ thick transparent conductive electrode coating of indium-tin-oxide (ITO) on glass. Finally, a flexible electrode (Au on polymer film) is placed on the top of the multilayered to make a contact. The particular device described here has three $\mathrm{Au}$ layers and two CdS layers, with 4 layers each of PAH and PSS as the inter-lying DL.

The film is insulating in the in-plane direction (6). In the vertical direction, the film is conducting due to tunneling between the Au and CdS layers. The current density, J, through the film as a function of bias, V, between the ITO and $\mathrm{Au}$ electrode, under a uniform pressure (i.e., compressive stress) $\sigma$, is shown in Fig. 2(a). The uniform pressure is applied by placing an optically flat quartz disk on the flexible Au electrode. The nonlinear J-V curve is fit based on a model combining the field assisted electron tunneling current (7) through the nanoparticles and the ionic (leakage) current due to ions in the polyelectrolyte. The total current (solid curve in Fig. 2(a)) is therefore modeled as,

$$
J=J_{T}+J_{I}=P \exp \left(-\frac{a K}{V}\right)+\frac{V}{R}
$$


In the first term due to tunneling, "a" is the (vertical) interparticle distance, " $\mathrm{K}$ " is a critical field for activated tunneling that depends on the work functions of the particles, and " $P$ " at constant temperature is proportional to $\mathrm{V}^{2}$ and the number density of carriers for conduction(7). The second term due to the mobile ions $\left(\mathrm{H}^{+}, \mathrm{Na}^{+}\right.$and $\left.\mathrm{OH}^{-}, \mathrm{Cl}^{-}\right)$in the polyelectrolytes, " $\mathrm{R}$ ", is the ohmic resistance that is proportional to the distance between the electrodes. The fits are performed as a two step process: (i) At low V, the current is dominated by ionic current. Thus, the slope of the linear region at low- $\mathrm{V}$ is $1 / \mathrm{R}$. The estimated resistivity of $\sim$ $1.6 \times 10^{8} \Omega-\mathrm{m}$ is reasonable for ion conductivity in PSS and PAH under ambient humidity. (ii) Thus, $J-(V / R)$ is $J_{T}$ that is fit by a single exponential to obtain $(\mathrm{Ka})$ and $\mathrm{P}$. Consistent with the theory on field-assisted tunneling, $\mathrm{P} \sim \mathrm{V}^{2}$ (Fig.2(b)). Assuming 'a' (at $\sigma=0) \sim 5 \mathrm{~nm}$, the critical field $\mathrm{K}$ of $\sim 10^{9}$ $\mathrm{V} / \mathrm{m}$ is reasonable. Because, electroluminescence will occur only when tunneling through $\mathrm{CdS}$ occurs,

electroluminescence intensity, $\mathrm{I}_{\mathrm{EL}}$, should be measurable above the bias of $\sim 8 \pm 1 \mathrm{~V}$ when the total $\mathrm{J}$ begins to increase beyond the straight line (i.e., $\mathrm{J}_{\mathrm{I}}$ ) (Fig.2(a)). Fig. 2(c) is consistent with electroluminescence initiation at $\sim 8 \pm 1 \mathrm{~V}$.

As the electroluminescence intensity from the device is virtually continuous (because inter-particle spacing is well below $25 \mathrm{~nm}$ ), the resolution of the stress image is determined by the optics and the CCD camera and the "smearing" of the stress due to finite thickness of the plastic backing for the Au-electrode. Using the current device the stress distribution image due to the embossing of "Ashok Chinha" on 5 Rupee coin of India is clearly visible by pressing the coin at a reasonable load (Fig. 2(d))(6). For the CCD with a $512 \times 512$ array of $\sim 16 \mu$ m pixels, the $\mathrm{I}_{\mathrm{EL}}$ is well over noise level to achieve lateral spatial resolution of at least $\sim 20 \mu \mathrm{m}$ and the height modulation of $\sim 10 \mu \mathrm{m}$ is measurable, indicating the device is fairly deformable (6). The linearity of the device is shown in Fig. 3(a). Both J and $\mathrm{I}_{\mathrm{EL}}$ increases linearly with increasing magnitude of the compressive stress. The linearity of the device is due to the dominance of ' $\mathrm{P}$ ' for modulation of tunneling current (Fig. 3(b)). Although, the tunneling process has an exponential dependence on ' $a$ ' that decreases with increasing $\sigma$ (eq. (1)), the linear change in ' $\mathrm{P}$ ' with load $(=\sigma)$ dominates the modulation of tunneling process (i.e., current). Physically, ' $\mathrm{P}$ ' is proportional to electron density of conduction electrons. At fixed temperature, the electron conduction density is constant. Thus, the increase in ' $\mathrm{P}$ ' is proportional to the increase in number of percolation channels between the electrodes - as load increases, the number density of percolating channels of nanoparticles traversing through the thickness increases linearly causing a linear increase in ' $\mathrm{P}$ '.

\section{SUMMARY AND CONCLUSION}

We have briefly discussed the characteristics of a thin film tactile device based on electron tunneling phenomena that converts applied stress to electroluminescent light. By focusing the electroluminescent light on a camera a high resolution stress-image is obtained. The tunneling current is modulated by altering the percolation behavior of the nanoparticle clusters leading to linear response.

\section{REFERENCES}

1. B. Gates, Scientific American, p. 58, January 2007.

2. R. Crowder, Science, 312, 1478 (2006).

3. T. Someya et al., Proc. Nat. Acad. Sci.101, 9966 (2004).

4. J. W. Morley, et al., Expt. Brain Research 49, 291 (1983).

5. G. Decher, Science 277, 1232 (1997).

6. V. Maheshwari, R.F. Saraf, Science, 312, 1501 (2006).

7. R. H. Fowler, et al., Proc. Royal Soc. A. 119, 173 (1928).
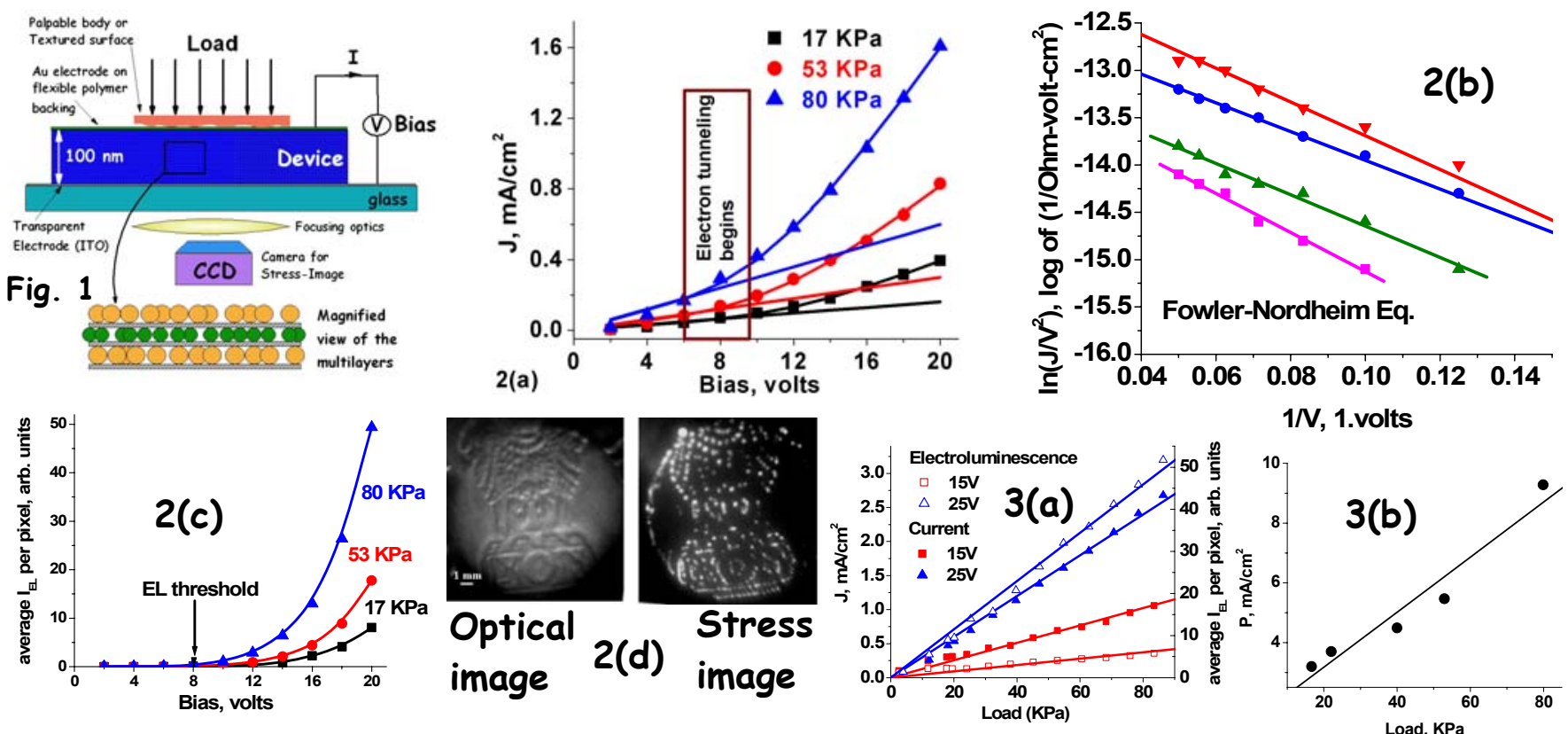